\title{
Symptom and Anxiety Assessment in Gynecologic Cancer Patients Receiving Chemotherapy
}

\author{
Pongsaton Viriyasiri, Phatthanan Phutthikiat, Phatthawan Phonmak, Phurinut \\ Krutjaikla, Sittichai Ongtip, Prapaporn Suprasert
}

Department of Obstetrics and Gynecology, Faculty of Medicine, Chiang Mai University, Thailand.

\begin{abstract}
Background: Side effects of chemotherapy usually disturbed the daily life of patients. During chemotherapy, quality of life of patients is affected by the severity of symptoms experienced. Objective: To evaluate the side effects experienced by gynecologic cancer patients receiving chemotherapy. Methods: Gynecologic cancer patients receiving chemotherapy (at least 1 cycle with standard premedication that included antiemetic drugs) between 18 June and 25 September 2019 were invited to this study. Participants were interviewed by our team for personal data and attitudes toward their disease and treatment. In addition, the Edmonton Symptom Assessment System (ESAS-Thai version) tool, and Multinational Association for Supportive Care in Cancer (MASCC) antiemetic tool were used to assess the symptoms during chemotherapy. Results: One hundred sixty-five participants entered this study. The mean age was 53.5. The three most common type of cancer were ovarian (37.6\%), cervical (37.6\%) and uterine cancer (21.8\%). Most common chemotherapy was carboplatin plus paclitaxel $(64.8 \%)$. Two-thirds of the participants believed they could be cured. The most common severe symptom from the ESAS tool was pain $(20.6 \%)$, followed by fatigue $(18.8 \%)$, appetite change $(16.4 \%)$ and numbness $(10.3 \%)$. In addition, $10.9 \%$ of patients experienced nausea/vomiting in acute phase, while $20.6 \%$ experienced it in the delayed phase. Conclusion: Our participants revealed positive attitudes toward cancer and treatment. Some patients experienced nausea and vomiting despite using antiemetic drugs. The most frequent self-reported symptom was pain. Therefore, pain control was necessary to improve their quality of life.
\end{abstract}

Keywords: Chemotherapy- gynecologic cancer- CINV- symptom assessment- ESAS- MASCC antiemetic tool

Asian Pac J Cancer Care, 5 (2), 95-100

\section{Introduction}

Chemotherapy is one of the main treatments in gynecologic cancer especially in advanced stages and recurrences. Various regimens such as Carboplatin plus Paclitaxel, Cisplatin plus 5 -Fluorouracil, Gemcitabine, etc. are frequently used [1]. It is known that these chemotherapy regimens cause many side effects like nausea and vomiting, anemia, neutropenia, numbness, etc. Both chemotherapy side effects and the symptoms of the cancer negatively impact the quality of life of the patients. Akin and Durna [2] conducted a comparative study of the symptoms occurring after receiving chemotherapy by using the Edmonton Symptom Assessment System (ESAS) in 119 patients receiving chemotherapy. The authors compared the ESAS answers among patients, family caregivers
Submission Date: 02/26/2020 Acceptance Date: 05/05/2020

\footnotetext{
Corresponding Author:

Dr. Prapaporn Suprasert

Department of Obstetrics and Gynecology, Faculty of Medicine, Chiang Mai University, Thailand.

Email: psuprase@gmail.com
}

and nurses and reported that the most frequent severe symptoms were tiredness, loss of well-being, anxiety, drowsiness, appetite change, depression, pain and nausea. In addition, the authors summarized that the patients and caregivers demonstrated a strong agreement regarding symptoms in contrast with patients and nurses that showed poor to fair agreement. Therefore, self-assessment by patients was important. Regarding gynecologic cancer, Nazik et al [3] studied anxiety and symptoms in Turkish gynecologic cancer patients receiving chemotherapy by using the ESAS and the State-Trait Inventory. They found that the average highest point of symptom was fatigue while the level of anxiety was moderate. Furthermore, chemotherapy-induced nausea and vomiting (CINV) 
was mentioned as one of the adverse events that affected the daily life of patients [4]. However, the data of the symptoms including CINV assessed by the patients in Thailand is still limited. Therefore, we conducted this study to evaluate these aspects of our gynecologic cancer patients during chemotherapy. We hope that the outcome should be beneficial for health-care providers to improve quality of life of those patients.

\section{Materials and Methods}

\section{Study design}

This study was designed as a cross-sectional survey and approved by the Research Ethics Committee 4, Faculty of Medicine, and Chiang Mai University. The study code is OBG-2562-06369.

\section{Patient selection}

The gynecologic cancer patients who received chemotherapy (at least 1 cycle) at the Gynecologic Oncology Unit, Chiang Mai University Hospital from 18 June 2019 to 25 September 2019 and were able to communicate in Thai were invited to participate in this study. The patients whose performance status was poor (ECOG criteria more than 3), unable to interview, or revealed a symptom of nausea / vomiting from other causes besides chemotherapy were not invited in this project. All patients received standard premedication that consisted of lorazepam, serotonin antagonist, dexamethasone and anti-histamine about 30 minutes before initiated chemotherapy.

\section{Instruments}

The data were collected using 3 parts of case record form consisting of patient data form, ESAS (Thai version) form [5], and Multinational Association for Supportive Care in Cancer (MASCC) antiemetic tool (MAT) form [6].

The patient data form includes of the demographic data, gynecologic cancer data and the attitude of the patient to their disease and treatment outcome. The ESAS scale was developed by Eduardo Bruera in 1991 with the purpose to improve patient care in oncology by evaluating patient's opinion about the severity of his/her symptoms [7]. The ESAS evaluates nine common symptoms in patients with cancer: pain, tiredness, nausea, depression, anxiety, drowsiness, appetite change, loss of well-being and shortness of breath. However, patients could report other problems in this instrument. The severity of each symptom was rated on a numerical scale from 0 to 10 , with 10 meaning the worst severity. After that, the score was categorized to 4 levels; no symptom if score equaled to 0 , mild if the score was in a range of 1-3 scores, moderate if the score was in a range of 4-6 scores and severe if the score was more than 7 scores [7]. In this study, we used ESAS Thai version that was validated with the original [5].

The third part of the data collection is the MAT. The MAT was first created and posted in 2004 by members MASCC of to assist patients and oncology professionals in communicating accurately about nausea and vomiting that may occur with chemotherapy. We used this tool by translating to Thai language to evaluate nausea and / or vomiting of the patients. The definition of vomiting was the bringing up of stomach contents and the definition of nausea was the feeling that you might vomit. The patients were asked about nausea and vomiting during the first 24 hours and at day 2 to day 4 after receiving the former chemotherapy. If vomiting was present, the patients were asked how many times it happened while if the nausea was presented, the patients were asked how much nausea was experienced during the first 24 hours and at day 2 to day 4 . Nausea and/or vomiting that occurred during 24 hours were classified as acute phase whereas the nausea and/or vomiting that occurred after that (day 2 - day 4) were classified as delayed phase.

The participants were interviewed face to face by our investigator team during waiting to see the doctors at out-patient-department of gynecologic oncology unit for the next course of chemotherapy. The interview time was about 20 minutes. The medical information of each participant was obtained from electronic patients files.

\section{Statistical analysis}

The sample size for this study was estimated from the data of a previous study. The study revealed fatigue as the most frequent symptom which was found in $90 \%$ of 41 participants [3]. The 95 inter-percentile reference intervals for calculation accommodated the possibility of a loss of follow up participants. Thus, this study required to enroll about 160 participants.

The statistical analysis of the data was conducted using the IBM SPSS Statistics for Windows program (version 22). Descriptive statistics were used to summarize patient characteristics. Chi-square or Fisher's Extract test was used for comparative analysis of the factors between acute and delayed nausea and vomiting. A p-value of $<0.05$ was suggested statistically significant.

\section{Results}

There were 165 participants entered to the present study. The clinical data was noted in Table 1 . The mean age was 53.4. Over $90 \%$ were Thai. About two-thirds of the participants were married and about $75 \%$ had children. Only $14.5 \%$ of the participants were government service. Nearly $70 \%$ of participants did not finish high school. The majority of our participants had an income of less than 10,000 baht per month (about 331 US dollars). Around $63 \%$ of the participants did not have any underlying disease. Regarding drug abuse, the participants revealed alcohol addicted, cannabis used and smoking at $6.1 \%$, $3.0 \%$, and $2.4 \%$, respectively. The top three-cancer types were ovarian, cervical and uterine cancer and nearly $60 \%$ were in stages 3 and 4 . The most common chemotherapy regimen was carboplatin plus paclitaxel. $62.4 \%$ of the participants were undergoing chemotherapy for the first time and about $70 \%$ had experienced more than 1 cycle at the interview time.

Regarding attitude to their cancer, nearly $60 \%$ of the participants believed that they could be cured but it took a long time while $24 \%$ believed that it was not to be 
Table 1. Distribution of Clinical Data Related to Gynecologic Cancer Patients

\begin{tabular}{|c|c|}
\hline & $\mathrm{N}(\%)$ \\
\hline Mean age + SD (year) & $53.4(12.8)$ \\
\hline \multicolumn{2}{|l|}{ Race } \\
\hline Thai & $149(90.3)$ \\
\hline Others & $16(9.7)$ \\
\hline \multicolumn{2}{|l|}{ Status } \\
\hline Single & $32(19.4)$ \\
\hline Married & $111(67.3)$ \\
\hline Divorce & $6(3.6)$ \\
\hline Widow & $16(9.7)$ \\
\hline \multicolumn{2}{|l|}{ Number of children } \\
\hline 0 & $42(25.5)$ \\
\hline 1 & $37(22.4)$ \\
\hline 2 & $63(38.2)$ \\
\hline 3 & $15(9.1)$ \\
\hline$>3$ & $8(4.6)$ \\
\hline \multicolumn{2}{|l|}{ Occupation } \\
\hline Government service & $24(14.5)$ \\
\hline State enterprise & $1(0.6)$ \\
\hline Merchant & $16(9.7)$ \\
\hline Employment & $24(14.5)$ \\
\hline Owner & $6(3.6)$ \\
\hline Others & $94(57)$ \\
\hline \multicolumn{2}{|l|}{ Education } \\
\hline Undergraduate & $115(69.7)$ \\
\hline Bachelor degree & $44(26.7)$ \\
\hline Postgraduate & $6(3.6)$ \\
\hline \multicolumn{2}{|l|}{ Income (per month: Baht) } \\
\hline$<10,000$ & $109(66.1)$ \\
\hline $10,000-20,000$ & $24(14.5)$ \\
\hline $20,000-30,000$ & $12(7.3)$ \\
\hline$>30,000$ & $20(12.1)$ \\
\hline No underlying disease & $105(63.6)$ \\
\hline Cannabis used & $5(3.0)$ \\
\hline Alcohol addicted & $10(6.1)$ \\
\hline Smoking & $4(2.4)$ \\
\hline \multicolumn{2}{|l|}{ Gynecologic cancer type } \\
\hline Ovary & $62(37.6)$ \\
\hline Cervix & $47(28.5)$ \\
\hline Corpus & $36(21.8)$ \\
\hline Fallopian tube & $10(6.1)$ \\
\hline Gestational Trophoblastic Neoplasia & $8(4.8)$ \\
\hline Primary peritoneum & $1(0.6)$ \\
\hline Vagina & $1(0.6)$ \\
\hline \multicolumn{2}{|l|}{ Stage } \\
\hline I & $35(21.2)$ \\
\hline II & $28(17.0)$ \\
\hline III & $74(44.8)$ \\
\hline IV & $28(17.0)$ \\
\hline
\end{tabular}

Continued Table 1.

\begin{tabular}{lc}
\hline & $\mathrm{N}(\%)$ \\
\hline Chemotherapy & $107(64.8)$ \\
Carboplatin plus Paclitaxel & $10(6.1)$ \\
Gemcitatin plus 5 -Fluorouracil & $8(4.8)$ \\
Weekly Paclitaxel & $5(3.0)$ \\
Others & $35(21.2)$ \\
Chemo naive & $103(62.4)$ \\
Current cycle of chemotherapy & \\
1 & $45(27.3)$ \\
2 & $29(17.6)$ \\
3 & $20(12.1)$ \\
4 & $21(12.7)$ \\
5 & $15(9.1)$ \\
6 & $32(19.4)$ \\
$>6$ & $3(1.8)$ \\
Phase of treatment & \\
$3-12$ months & $105(63.6)$ \\
$>12$ months & $60(36.4)$ \\
\hline
\end{tabular}

cured. Concerning the attitude of chemotherapy, over $90 \%$ believed that chemotherapy could cure cancer and half of them were concerned about the side effect. Interestingly, there was no significant difference between the line of chemotherapy and the patients' attitude of cancer as presented in Table 2.

About the ESAS outcome, Figure 1A and 1B showed the number of patients categorized by the degree of each symptom and the rank of severity of symptoms. The numbness was the symptom that patients frequently complained about. Thus, we included this symptom in part of the interview later. $20.6 \%$ of the patients identified pain as the most frequent severe symptom, fatigue (18.8\%), appetite change (16.4\%) and numbness (10.3\%). Regarding severe numbness in 16 patients, the most two frequent chemotherapy regimens were Carboplatin plus Paclitaxel (9 cases) and Cisplatin plus 5-Fluorouracil (4 cases).

Concerning CINV from the participants' view, in the acute phase, $18.2 \%$ experienced vomiting with the median episode at 2 (range 1-8) times and $25.5 \%$ experienced nausea with a median scale at 3.5 (range 1-8). Moreover, in delayed phases, $26.1 \%$ experienced vomiting with the median episode at 3 (range of 1-10) times and $45.5 \%$ experienced nausea with the median scale at 5 (range 1-10). Table 3 showed the relation between CINV in acute and delayed phases and found that there was a significant difference in both phases. Patients who experienced vomiting and/or nausea in both acute and delayed phases were $10.9 \%$ and $20.6 \%$, respectively. 


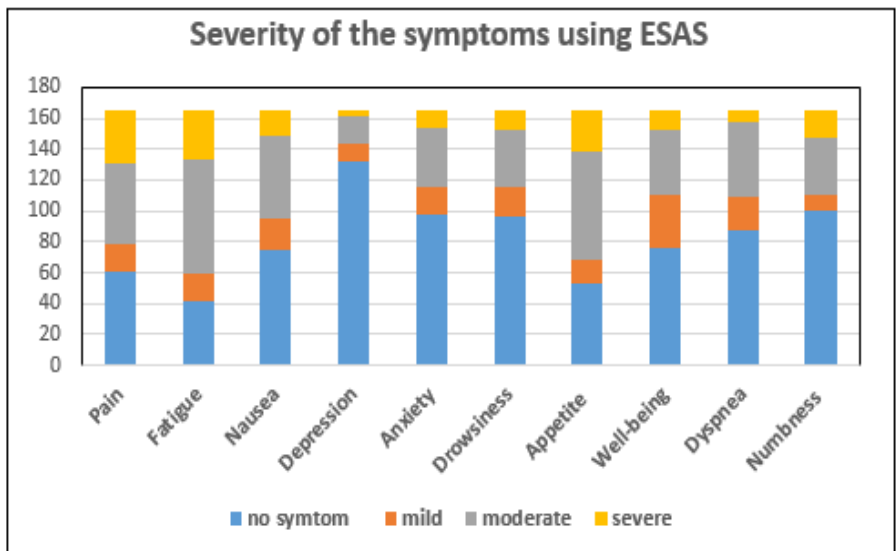

1A, Severity of the Symptoms Divided to Four Levels

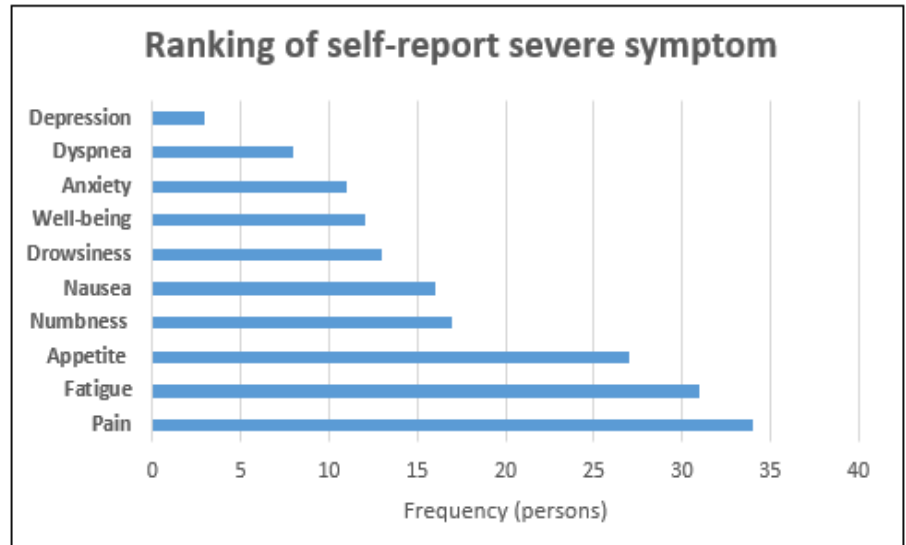

1B, Ranking of Self-report Severe Symptom

Figure 1. Self- assessment Using the Edmonton Symptom Assessment System (ESAS)

\section{Discussion}

This study revealed a positive attitude to cancer from our participants. Over $90 \%$ of the chemonaive and recurrent setting believed that their cancer was cured with chemotherapy. This was unlike the following report by Badihian et al.[8] who conducted a cross-sectional survey among 953 non-institutionalized individuals in Isfahan, Iran from November 2014 to February 2015 and found about one-third of participants agreed that it is very hard to regain health after a diagnosis of cancer even when treated with highly developed medical science. However, our data came from the participants who developed cancer and still received chemotherapy with the hope to cure. It was noticed that about $3 \%$ of our participants used cannabis during chemotherapy with the reason to help them sleep. The benefits and harms of cannabis to cancer are inconclusive due to inconsistent findings and lacked well design evidence [9].

Regarding CINV, one-third of the participants revealed vomiting and half of the participant's revealed nausea that occurred in the acute and/or delayed phase. Despite

Table 2. Attitude to Cancer Divided by Chemotherapy Status $(\mathrm{N}=165)$

\begin{tabular}{|c|c|c|c|c|}
\hline & \multicolumn{2}{|c|}{ Chemotherapy status $(\mathrm{N})$} & \multirow[t]{2}{*}{ Total } & \multirow[t]{2}{*}{ P value } \\
\hline Disease & First line $(\%)$ & Beyond first line (\%) & & \\
\hline The disease could not be cure & $24(14.5)$ & $16(9.7)$ & $40(24.2)$ & $0.258 *$ \\
\hline The disease took a long time to cure & $57(34.5)$ & $39(23.6)$ & $96(58.2)$ & \\
\hline The disease was easy to cure & $22(13.3)$ & $7(4.2)$ & $29(17.6)$ & \\
\hline Total & $103(62.4)$ & $62(37.6)$ & $165(100)$ & \\
\hline \multicolumn{5}{|l|}{ Chemotherapy } \\
\hline It cured cancer & $43(26.1)$ & $24(14.5)$ & $67(40.6)$ & $0.197 \#$ \\
\hline It cured cancer with side effect & $57(34.5)$ & $32(19.4)$ & $89(53.9)$ & \\
\hline It cannot cure cancer & $3(1.8)$ & $6(3.6)$ & $9(5.5)$ & \\
\hline Total & $103(62.4)$ & $62(37.6)$ & $165(100)$ & \\
\hline
\end{tabular}

*Chi-square test; \#Fisher exact test 
Table 3. The relation between Acute and Delayed Phase of Nausea and Vomiting

\begin{tabular}{lcccc}
\hline Acute phase & \multicolumn{2}{c}{ Delayed phase } & Total & P value* \\
& Yes (\%) & No (\%) & $<0.001$ \\
\hline Vomiting & $18(10.9)$ & $12(7.3)$ & $30(18.2)$ & \\
Yes & $25(15.2)$ & $110(66.7)$ & $135(81.8)$ & \\
No & $43(26.1)$ & $122(73.9)$ & $165(100)$ & $<0.001$ \\
Total & & & \\
Nausea & $34(20.6)$ & $8(4.8)$ & $123(74.5)$ & $165(100)$ \\
Yes & $41(24.8)$ & $82(49.7)$ &
\end{tabular}

the antiemetic drugs, CINV still occurred. However, our result was similar to the previous study from Hsieh et al [10]. They studied the incidence of CINV after highly or moderately emetogenic chemotherapy for cancer in over 600 patients from 6 Asia Pacific countries using MASCC antiemetic tool like us. The authors reported emesis and nausea was observed in $30 \%$ and $50 \%$ both acute and delayed phase, respectively. They also found that physicians tended to underestimate the nausea rate especially in the delayed phase but overestimated emesis incidence. The underestimation of nausea was still the problem and it was difficult to control with standard antiemetic drug like serotonin-antagonist [11]. CINV was associated with a negative effect on daily life including effects on food intake, weight loss, effects on social interaction, dehydration, difficulty with sleeping and anxiety [4].

Concerning self-assessment with the ESAS system, our study found the most frequent severe symptom was pain followed by fatigue, appetite change, and numbness. This finding was different from Nazik et al [3]. The authors using the ESAS tool for assessment 41 Turkish gynecologic cancer patients receiving chemotherapy at least 3 cycles and showed the most frequent severe symptom was fatigue followed by drowsiness, depression, and pain. The difference might be from the distinction of nationality and the number of participants. However, all severe symptoms that disturbed participants were reversible except numbness. In our study, numbness was the symptom that we included later due to many patients complaints about this symptom in the initial phase of the survey. Hence, the rate of severe numbness in our study was underestimated. Numbness is one kind of neurotoxicity frequently developed form receiving cisplatin and paclitaxel and no effective treatment to improve [12].

The strength of the present study was a sufficient number of participants in one institution. Thus, the guideline of treatment was similar. In addition, our study was interviewed by well-trained interviewers. Thus, the data was more reliable than the participants self -report. However, this study recruited about one-fourth of the participants who just received only 1 cycle of chemotherapy. Therefore, they might not be represented as cumulative symptoms.
For future research, we recommended conducting the study with in more specific chemotherapy regimens. Accordingly, the management of side-effects in a specific regimen could be improved.

In conclusion, our patients revealed a positive aspect to cancer and treatment. A part of patients still observed nausea and vomiting even using the anti-emesis drugs. The most frequent self- report symptom was pain. Hence, pain control should be a significant part of any treatment in order to improve quality of life.

\section{Acknowledgements}

We received the funding from Faculty of Medicine, Chiang Mai University. We would like to thanks the nurses at Outpatient Department and Oncologic site at Maharaj Nakorn Chiang Mai Hospital; Miss Sukanya Yanunto, Miss Sopida Fanchomphu and Miss Orathai Baisai for their supports and Chiang Mai University English Language Team (CELT) consultant for providing language help.

\section{Conflict of Interest Statement}

The authors have no conflicts of interest to disclose.

\section{References}

1. NCCN Clinical Practice Guidelines in Oncology; 2019 [2019 Dec 31]. Available from: https://www.nccn.org/store/Profile/ Profile.aspx?requiredid $=1$

2. Akin S, Durna Z. A comparative descriptive study examining the perceptions of cancer patients, family caregivers, and nurses on patient symptom severity in Turkey. Eur J Oncol Nurs 2013;17:30-7.

3. Nazik E, Arslan S, Nazik H, Narin MA, Karlangic H, Koc Z. Anxiety and symptom assessment in Turkish gynecologic cancer patients receiving chemotherapy. Asian Pac J Cancer Prev. 2012;13:3129-33.

4. Salihah N, Mazlan N, Lua PL. Chemotherapy-induced nausea and vomiting: exploring patients' subjective experience. J Multidiscip Healthc 2016;9:145-51.

5. Chinda M, Jaturapatporn D, Kirshen AJ, Udomsubpayakul U. Reliability and validity of a Thai version of the edmonton symptom assessment scale (ESAS-Thai). J Pain Symptom Manage $2011 ; 42: 954-60$.

6. MASCC antiemesis tool (MAT).[2019 Dec 31]. Available 
from https://www.mascc.org/assets/Guidelines-Tools / mat_english_questionnaire_2010_2.pdf

7. Bruera E, Kuehn N, Miller MJ, Selmser P, Macmillan K. The Edmonton Symptom Assessment System (ESAS): a simple method for the assessment of palliative care patients. J Palliat Care 1991;7:6-9.

8. Badihian S, Choi EK, Kim IR, Parnia A, Manouchehri N, Badihian N,et al. Attitudes Toward Cancer and Cancer Patients in an Urban Iranian Population. Oncologist 2017;22:944-50.

9. Pratt M, Stevens A, Thuku M, Butler C, Skidmore B, Wieland LS,et al. Benefits and harms of medical cannabis: a scoping review of systematic reviews. Syst Rev 2019;8:320.

10. Hsieh RK, Chan A, Kim HK, Yu S, Kim JG, Lee MA,et al. Baseline patient characteristics, incidence of CINV, and physician perception of CINV incidence following moderately and highly emetogenic chemotherapy in Asia Pacific countries. Support Care Cancer 2015;23:263-72.

11. Shankar A, Roy S, Malik A, Julka PK, Rath GK. Prevention of Chemotherapy-Induced Nausea and Vomiting in Cancer Patients. Asian Pac J Cancer Prev 2015;16:6207-13.

12. Teoh D, Smith TJ, Song M, Spirtos NM. Care After Chemotherapy: Peripheral Neuropathy, Cannabis for Symptom Control, and Mindfulness. Am Soc Clin Oncol Educ Book 2018;38:469-79.

\section{(i) (3)}

This work is licensed under a Creative Commons AttributionNon Commercial 4.0 International License. 